\title{
ON THE DEGREE OF APPROXIMATION TO A HARMONIC FUNCTION*
}

\section{BY J. L. WALSH}

It is well known that if a function $u(x, y)$ is harmonic in a closed Jordan region of the $(x, y)$-plane, then in that closed region, $u(x, y)$ can be expanded in a uniformly convergent series of harmonic polynomials in $(x, y)$. A more explicit result can be proved, however, and it is the object of the present note to establish such a more general theorem.

TheOREM I. Let $C$ be an arbitrary closed Jordan region of the $(x, y)$-plane, and let $w=\phi(z), z=x+i y$, be a function which maps conformally the exterior of $C$ onto the exterior of the unit circle in the w-plane so that the points at infinity correspond to each other. Let $C_{R}$ denote the curve $|\phi(z)|=R, R>1$, that is, the transform onto the $z$-plane of the circle $|w|=R$.

$A$ necessary and sufficient condition that an arbitrary function $u(x, y)$, defined in $C$, be harmonic in (the closed region) $C$ is that there should exist harmonic polynomials $p_{n}(x, y)$ of degree $n, \dagger n=0,1,2, \cdots$, and numbers $M, R>1$, such that the inequalities

$$
\mid u(x, y)-p_{n}\left(x, y \mid \leqq \frac{M}{R^{n}},\right.
$$

where $M$ and $R$ are constants, that is, independent of $n$ and of $x, y$, and where $R>1$, should be valid for every point $(x, y)$ in $C$.

If the polynomials $p_{n}(x, y)$ are given so that $(1)$ is satisfied for every $(x, y)$ in $C$, the sequerice $\left\{p_{n}(x, y)\right\}$ converges everywhere interior to $C_{R}$ and uniformly on any closed point set

* Presented to the Society, September 9, 1926.

$\dagger$ A harmonic polynomial of degree $n$ is a harmonic polynomial of the form $\sum_{p+q \leqq n} a_{p q} x^{p} y^{q}$. No assumption of the non-vanishing of the $a_{p q}$ is made. Compare, however, the discussion given in the paper referred to in the second following footnote. A similar remark applies to the degree of $V_{n}(z)$. 
interior to $C_{R}$, so the function $u(x, y)$ is harmonic throughout the interior of $C_{R}$.*

If $u(x, y)$ is given harmonic in the closed region interior to $C_{\rho}$, the polynomials $p_{n}(x, y)$ can be chosen to satisfy (1) with $R=\rho$, for $(x, y)$ in $C$.

Theorem $I$ is analogous to, and will be proved by means of, the following theorem. $\dagger$

THEOREM II. Let $C$ be an arbitrary closed Jordan region of the z-plane, and let $w=\phi(z)$ be a function which maps conformally the exterior of $C$ onto the exterior of the unit circle in the w-plane so that the points at infinity correspond to each other. Let $C$ denote the curve $|\phi(z)|=R, R>1$, that is, the transform onto the z-plane of the circle $|w|=R$.

$A$ necessary and sufficient condition that an arbitrary function $F(z)$ defined in $C$ be analytic in the closed region $C$ is that there should exist polynomials $V_{n}(z)$ of degree $n, n=0$, $1,2, \cdots$, and numbers $M, R>1$, such that the inequalities

$$
\left|F(z)-V_{n}(z)\right| \leqq \frac{M}{R^{n}}
$$

where $M$ and $R$ are constants, that is, independent of $n$ and of $z$, and where $R>1$, are valid for every $z$ in $C$.

If the polynomials $V_{n}(z)$ are given so that (2) is satisficd, the sequence $\left\{V_{n}(z)\right\}$ converges everywhere interior to $C_{R}$ and uniformly on any closed point set interior to $C_{R}$, and thus $F(z)$ is analytic throughout the interior of $C_{R}$.

* Here and below we tacitly assume that if $u(x, y)$ is not originally supposed to be defined on the entire point set considered, then the definition in the new points is to be made by harmonic extension-or what amounts to the same thing, by means of the convergent series of harmonic polynomials. A similar remark applies in Theorem II.

$\dagger$ The names of S. Bernstein, M. Riesz, Faber, Fejér, Szegö should be particularly mentioned in connection with Theorem II. Proof of that theorem and detailed references to the literature are to be found in a paper by the present writer, Sitzungsberichte der Bayerischen Akademie der Wissenschaften, 1926, pp. 223-229. Compare also a forthcoming paper by Szegö in the same journal. 
If $F(z)$ is given analytic in the closed region interior to $C_{\rho}$, the polynomials $V_{n}(z)$ can be chosen so that (1) is satisfied for $R=\rho$ and $z$ in $C$.

If the function $u(x, y)$ of Theorem $\mathrm{I}$ is given harmonic in the closed region interior to $C_{\rho}$, the function

$$
F(z)=u(x, y)+i v(x, y), \quad z=x+i y,
$$

where $v(x, y)$ is a function conjugate to $u(x, y)$, is analytic likewise in the closed region interior to $C_{\rho}$, and hence the polynomials $V_{n}(z)$ exist so that inequalities (2) are satisfied for $z$ in $C$ and for $R=\rho$. The real part of $F(z)-V_{n}(z)$ is $u(x, y)-p_{n}(x, y)$, where $p_{n}(x, y)$ is a harmonic polynomial of degree $n$, so inequalities (1) are satisfied, $R=\rho,(x, y)$ in $C$.

It remains merely to prove that part of Theorem I concerning itself with given polynomials $p_{n}(x, y)$. Here we find it convenient to prove two lemmas.

LEMmA I. Let $\Gamma$ be an arbitrary analytic Jordan curve in the $(x, y)$-plane and $\Gamma^{\prime}$ an analytic Jordan curve interior to $\Gamma$. Then there exists a constant $\gamma$ depending only on $\Gamma$ and $\Gamma^{\prime}$ such that the inequality

$$
\left|u^{\prime}(x, y)\right| \leqq \mu,
$$

where $(x, y)$ is in or on $\Gamma$, and where $u^{\prime}(x, y)$ is an arbitrary function harmonic in the closed interior of $\Gamma$, implies the inequalities

$$
\left|\frac{\partial u^{\prime}(x, y)}{\partial x}\right| \leqq \gamma \mu,\left|\frac{\partial u^{\prime}(x, y)}{\partial y}\right| \leqq \gamma \mu,
$$

where $(x, y)$ is in or on $\Gamma^{\prime}$.

The proof of Lemma I is easy, by Green's formula:

$$
u^{\prime}(x, y)=\frac{1}{2 \pi} \int_{\Gamma} u^{\prime}(\xi, \eta) \frac{\partial g(\xi, \eta ; x, y)}{\partial n} d s
$$

where $g$ denotes the Green function for the region interior to $\Gamma$, and $n$ the inner normal. Partial differentiation of (5) 
with respect to $x$ and with respect to $y$, if in the right-hand member we differentiate under the integral sign, yields inequalities (4), where $\gamma$ is the larger of the upper bounds of the two quantities

$$
\frac{1}{2 \pi} \int_{\Gamma}\left|\frac{\partial^{2} g(\xi, \eta ; x, y)}{\partial x \partial n}\right| d s, \quad \frac{1}{2 \pi} \int_{\Gamma}\left|\frac{\partial^{2} g(\xi, \eta ; x, y)}{\partial y \partial n}\right| d s .
$$

These two quanitities are of course bounded when $(x, y)$ lies (as we here suppose) on or within $\Gamma^{\prime}$.

Lemma I will be ued in proving the following lemma.

Lemma II. Let $\Gamma$ be an analytic Jordan curve in the $(x, y)$ plane and $\Gamma^{\prime}$ an analytic Jordan curve interior to $\Gamma$. Then there exists a constant $\gamma^{\prime}$ depending only on $\Gamma$ and $\Gamma^{\prime}$ such that the inequality (3), $\left|u^{\prime}(x, y)\right| \leqq \mu$, where $(x, y)$ is in or on $\Gamma$ and where $u^{\prime}(x, y)$ is an arbitrary function harmonic in the closed interior of $\Gamma$, implies the existence of a function $v^{\prime}(x, y)$ conjugate to $u^{\prime}(x, y)$ on and interior to $\Gamma$ such that

$$
\left|v^{\prime}(x, y)\right| \leqq \gamma^{\prime} \mu
$$

where $(x, y)$ is in or on $\Gamma^{\prime}$.

Denote by $(a, b)$ any fixed point interior to $\Gamma^{\prime}$; then the lengths of the shortest curves in the closed interior of $\Gamma^{\prime}$ from $(a, b)$ to the various points in and on $\Gamma^{\prime}$ have a finite upper limit $L .^{*}$ If we introduce the definition

$$
\begin{aligned}
v^{\prime}(x, y)=\int_{(a, b)}^{(x, y)}\left(-\frac{\partial u^{\prime}}{\partial y} d x+\frac{\partial u^{\prime}}{\partial x} d y\right)= & \int_{(a, b)}^{(x, y)} \frac{\partial u^{\prime}}{\partial n} d s, \\
& (x, y \text { in or on } \Gamma),
\end{aligned}
$$

where the normal $n$ has the same relation to the direction $s$ as the positive axis of $y$ to the positive axis of $x$, the function $v^{\prime}(x, y)$ is conjugate to $u^{\prime}(x, y)$ in and on $\Gamma$. This integral is independent of the path of integration in $\Gamma$; we take, then, the integral over the shortest path in $\Gamma$ from $(a, b)$ to $(x, y)$, when $(x, y)$ lies in or on $\Gamma^{\prime}$. Inequalities (4) give us

\footnotetext{
* The reader should have no difficulty in proving this statement.
} 


$$
\left|\frac{\partial u^{\prime}}{\partial n}\right| \leqq 2^{1 / 2} \gamma \mu
$$

so we have, if $(x, y)$ is in or on $\Gamma^{\prime},\left|v^{\prime}(x, y)\right| \leqq 2^{1 / 2} \mu \gamma L$, which is of the form (6).

We return now to the proof of Theorem $I$. Let $\Gamma$ be an analytic Jordan curve interior to $C$, and $\Gamma^{\prime}$ an analytic Jordan curve interior to $\Gamma$. If the harmonic polynomials $p_{n}(x, y)$ satisfying (1) are given, we have

$$
\begin{gathered}
\left|u(x, y)-p_{n}(x, y)\right| \leqq \frac{M}{R^{n}}, \quad \mid u(x, y)-p_{n-1}\left(x, y \mid \leqq \frac{M}{R^{n-1}},\right. \\
\left|p_{n}(x, y)-p_{n-1}(x, y)\right| \leqq M \frac{1+R}{R^{n}},
\end{gathered}
$$

where $(x, y)$ is in or on $\Gamma$. There exists therefore a function $q_{n}(x, y)-q_{n-1}(x, y)$ defined in and on $\Gamma$ and conjugate to $p_{n}(x, y)-p_{n-1}(x, y)$ such that we have by (6)

$$
\left|q_{n}(x, y)-q_{n-1}(x, y)\right| \leqq \gamma^{\prime} M \frac{1+R}{R^{n}},
$$

where $(x, y)$ is in or on $\Gamma^{\prime}$. If we set $V_{n}(z)=p_{n}(x, y)$ $+i q_{n}(x, y)$, then $V_{n}(z)$ is a polynomial in $z$ of degree $n$ and we have

$$
\left|V_{n}(z)-V_{n-1}(z)\right| \leqq M \frac{\left(1+\gamma^{\prime}\right)(1+R)}{R^{n}},
$$

where $z$ is in or on $\Gamma^{\prime}$. It follows that the sequence $\left\{V_{n}(z)\right\}$ converges in and on $\Gamma^{\prime}$ to a function $F(z)$, and in such a way that we have

$$
\begin{aligned}
& \begin{aligned}
\left|F(z)-V_{n}(z)\right|= & \mid\left[V_{n+1}(z)-V_{n}(z)\right] \\
& +\left[V_{n+2}(z)-V_{n+1}(z)\right]+\cdots \mid
\end{aligned} \\
& \leqq M\left(1+\gamma^{\prime}\right)(1+R)\left[\frac{1}{R^{n+1}}+\frac{1}{R^{n+2}}+\cdots\right] \\
& =\frac{M\left(1+\gamma^{\prime}\right)(1+R)}{(1-R) R^{n+1}}, \quad\left(z \text { in or on } \Gamma^{\prime}\right),
\end{aligned}
$$


which is of form (2). Hence by Theorem II, the sequences $\left\{V_{n}(z)\right\}$ and $\left\{p_{n}(x, y)\right\}$ converge interior to $C_{R}^{\prime}$, and uniformly on any closed point set interior to $C_{R}{ }^{\prime}$, where $C_{R}{ }^{\prime}$ is the transform onto the $z$-plane of the circle $|w|=R$, when the exterior of the unit circle in the w-plane is mapped onto the exterior of $\Gamma^{\prime}$ in the $z$-plane so that the points at infinity correspond to each other.

But $\Gamma^{\prime}$ is really an arbitrary analytic Jordan curve interior to $C$, and can be chosen as near to the boundary of $C$ as we please. When $\Gamma^{\prime}$ approaches the boundary of $C$ uniformly, it follows from the classical results of Carathéodory* on conformal mapping that the curve $C_{R}^{\prime}$ approaches the curve $C_{R}$ uniformly, so Theorem I is established.

Theorem II is valid under a more general assumption than that stated, namely when instead of the closed interior of a Jordan curve we consider $C$ to be an arbitrary closed limited point set not a single point whose complementary set, with respect to the entire plane, is simply connected. We cannot extend Theorem I to this most general case, but can nevertheless prove a more general result than Theorem $I$.

If $C$ is an arbitrary limited region, simply or multiply connected, there may be points $P$, not belonging to the closed region $C$, which cannot be joined to the point at infinity by means of a simple arc containing no point of the closed region $C$. These points $P$ make up a finite or infinite number of regions $G$ whose boundary points are all points of the boundary of $C$. Thus if a sequence of harmonic polynomials or

* Mathematische Annalen, vol. 27 (1912), pp. 107-144, especially pp. 126-127. It is convenient to think of the curve $\Gamma^{\prime}$ as approaching the boundary of $C$ monotonically - that is, each successive curve $\Gamma^{\prime}$ lies exterior to the preceding, so that the curves $C_{R}{ }^{\prime}$ likewise vary monotonically, and hence $C_{R}{ }^{\prime}$ approaches $C_{R}$ uniformly. For the details connected with nonmonotonic approach, see Carathéodory, loc. cit.

Here we may also apply the results of Courant, Göttinger Nachrichten, 1914, pp. 101-109; 1922, pp. 69-70, or of Lebesgue, Rendiconti di Palermo, vol. 24 (1907), pp. 371-402, especially pp. 398-399. But the results of Caratheodory (his Satz V, together with the remark in $\$ \$ 12 \mathrm{a}$ and 19 on uniform convergence) are in precisely the proper form for our application. 
polynomials in $z$ converges uniformly in the closed region $C$, that sequence converges uniformly likewise in each of the closed regions $G$, for it converges uniformly on the boundary of every such region.

Such a sequence of harmonic polynomials may, however, represent different monogenic harmonic functions in $C$ and in a region $G$. We give here an illustration without proof. Let $C$ be a strip, closed at one end, that winds infinitely many times about the unit circle and approaches that circle. The circumference of the unit circle then belongs to the closed region $C$ and the interior of that circle is a region $G$. The function $u(x, y)=\log \left(x^{2}+y^{2}\right)^{1 / 2}$ is harmonic in the closed region $C$ and in that closed region may be represented by a uniformly convergent series of harmonic polynomials. ${ }^{*}$ This series of polynomials converges uniformly on the unit circle, but interior to that circle represents not $\log \left(x^{2}+y^{2}\right)^{1 / 2}$, but the function which is harmonic interior to that circle and takes on the values $\log \left(x^{2}+y^{2}\right)^{1 / 2}$ on the circumference, namely zero.

Let us in the general case call the extended interior of $C$ the closed point set $\bar{C}$ which is the totality of all points $P$ which cannot be joined to the point at infinity by a simple arc containing no point of the closed region $C$. We shall then prove a theorem which is particularly interesting in view of the illustration just given.

THEOREM III. Let $C$ be an arbitrary limited region of the $(x, y)$-plane, and let $B$ be the complementary set (with respect to the entire plane) of $\bar{C}$, the extended interior of $C$. Denote by $w=\phi(z), z=x+i y$, a function which maps $B$ conformally onto the exterior of the unit circle in the w-plane so that the points at infinity correspond to each other, and denote by $C_{R}$ the curve $|\phi(z)|=R, R>1$, that is, the transform onto the z-plane of the circle $|w|=R$.

\footnotetext{
* See a forthcoming paper by the present writer in Journal für Mathematik.
} 
$A$ necessary and sufficient condition that a function $u(x, y)$ defined on $\bar{C}$ (or in the closed region $C$ ) be harmonic on $\bar{C}$ is that there shall exist harmonic polynomials $p_{n}(x, y)$ of degree $n$, $n=0,1,2, \cdots$, and numbers $M$ and $R>1$, such that inequalities (1) are valid for all points $(x, y)$ of $\bar{C}$ (or of the closed region $C)$.

If the polynomials $p_{n}(x, y)$ are given so that (1) is satisfied for all points $(x, y)$ on $\bar{C}$ (or all points $(x, y)$ in the closed region $C)$, the sequence $\left\{p_{n}(x, y)\right\}$ converges everywhere interior to $C_{R}$ and uniformly on any closed point set interior to $C_{R}$, so the function $u(x, y)$ is harmonic everywhere interior to $C_{R}$.

If $u(x, y)$ is given harmonic in the closed region interior to $C_{\rho}$, the polynomials $p_{n}(x, y)$ can be chosen to satisfy (1) with $R=\rho$, for $(x, y)$ on $\bar{C}$.

If the function $u(x, y)$ is given harmonic in the closed interior of $C_{\rho}$, a conjugate function $v(x, y)$ exists which is likewise harmonic in that closed region. By the extension of Theorem II, the polynomials $V_{n}(z)$ can be found so that (2) is satisfied for $z$ in $\bar{C}$ and for $R=\rho$, where $F(z)=u(x, y)+i v(x, y)$. The real part $p_{n}(x, y)$ of the polynomial $V_{n}(z)$ is such that (1) is satisfied for $R=\rho$ and for $(x, y)$ in $\bar{C}$.

Suppose finally that the polynomials $p_{n}(x, y)$ are given so that (1) is satisfied for $(x, y)$ in the closed region $C$. The reasoning already used is then valid, even if $C$ is multiply connected, if a sequence $\Gamma_{n}^{\prime}$ of curves $\Gamma^{\prime}$ is chosen, the curve $\Gamma_{n}^{\prime}$ lying interior to $C$, every curve $\Gamma_{n}^{\prime}$ containing all points on and interior to $\Gamma_{n-1}^{\prime}$, every point interior to $C$ likewise interior to some $\Gamma_{n}^{\prime}$. For application of the results of Carathéodory it is sufficient to notice that no region $B^{\prime}$ containing $B$ but different from $B$ lies exterior to all the curves $\Gamma_{n}{ }^{\prime}$. Any such region $B^{\prime}$ would contain at least one interior point of either $C$ or a region $G$. Such a point cannot, however, be joined to the point at infinity by a curve containing no point of the boundary of $C$, hence cannot be joined to the point at infinity by a curve lying wholly in the open region $B^{\prime}$.

HARVARD UNIVERSITY 